\title{
Peltier effect in normal metal-insulator-heavy fermion metal junctions
}

\author{
A. V. Goltseva) \\ Ioffe Physico-Technical Institute, 194021 St. Petersburg, Russia \\ D. M. Rowe, V. L. Kuznetsov, L. A. Kuznetsova, and Gao Min \\ NEDO Laboratory for Thermoelectric Engineering, Cardiff University, United Kingdom
}

(Received 12 August 2002; accepted 7 February 2003)

\begin{abstract}
A theoretical study has been undertaken of the Peltier effect in normal metal-insulator-heavy fermion metal junctions. The results indicate that, at temperatures below the Kondo temperature, such junctions can be used as electronic microrefrigerators to cool the normal metal electrode and are several times more efficient in cooling than the normal metal-heavy fermion metal junctions.

(C) 2003 American Institute of Physics. [DOI: 10.1063/1.1565505]
\end{abstract}

The attention of physicists has been drawn to heavy fermion (HF) compounds, ${ }^{1,2}$ the unusual properties of which make them attractive for various physical applications such as thermoelectric devices. ${ }^{3-5}$ In this letter, the Peltier effect of a normal metal $(\mathrm{N})$-insulator $(\mathrm{I})-\mathrm{HF}$ metal $\left(\mathrm{N}_{\mathrm{HF}}\right)$ junction is investigated. It is shown that the $\mathrm{NIN}_{\mathrm{HF}}$ junction can be used as an electronic microrefrigerator. The physical principle of the microrefrigerator is that the current flowing through the junction removes high-energy thermal electrons from the normal metal, thus cooling it. Recently, electronic microrefrigerators based on normal metal-insulatorsuperconductor (NIS) tunnel junctions have been proposed and investigated. ${ }^{6,7}$ It is the existence of a superconducting gap in the superconductive electrode of the NIS junction that permits manipulation of the Fermi-Dirac distribution of electrons. Similar refrigeration effects also can be achieved through the internal field emission in a thin-film device, ${ }^{8}$ vacuum devices, ${ }^{9,10}$ and superconductor-semiconductorsuperconductor (SSS) structures. ${ }^{11}$

In the present letter, it is shown that the existence of a hybridization gap produced by strong electron correlations near the Fermi surface in a HF metal also allows manipulation of the energy transfer from the electron gas in the normal metal electrode (thermoelement) to electrons in the HF electrode in the NIN $_{\mathrm{HF}}$ junction. Calculation of the temperature dependence of the power transfer from the normal metal electrode reveals that it attains a maximum at a temperature below the Kondo temperature $T_{k}$.

The tunneling current and thermal properties of the $\mathrm{NIN}_{\mathrm{HF}}$ junction shown in Fig. 1(a) is investigated. A potential $V$ is applied to the normal metal electrode. The tunneling current is given by

$$
\begin{aligned}
I= & 2 \pi e \sum_{\nu \alpha k, \sigma p}\left|T_{\nu \alpha k, \sigma p}\right|^{2} \delta\left(\epsilon_{L p}+e V-E_{\nu k}\right) \\
& \times\left[f\left(\epsilon_{L p}\right)-f\left(E_{\nu k}\right)\right],
\end{aligned}
$$

where $\epsilon_{L p}$ is the kinetic energy of conduction electrons with momentum $p$ in the normal metal electrode, $f(E)$ is the Fermi-Dirac distribution function, $T_{\nu \alpha k, \sigma p}=\langle\nu \alpha k|H| \sigma p\rangle$ is the matrix element of the electron transition through the bar-

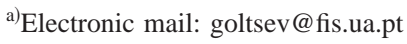

rier from an electron state $(\sigma p)$ in the normal metal electrode into a quasiparticle state $(\nu \alpha k)$ in the energy band $E_{\nu}(\mathbf{k})$ of the HF metal, $\sigma$ and $\alpha$ are spin indices, and $H$ is the Hamiltonian of the junction.

The thermal transport properties of the $\mathrm{NIN}_{\mathrm{HF}}$ junction are determined by the energy transferred by the tunneling current. When electrons with kinetic energy $\epsilon_{L p}$ are removed from the normal metal and transit into the HF metal, then electrons with an average kinetic energy equal to the chemical potential $\mu$ are returned to the normal metal electrode through normal contact. Then, the power transfer $P$ from the normal metal electrode on the left into the HF electrode on the right is given by

$$
\begin{aligned}
P= & 2 \pi \sum_{\nu \alpha k, \sigma p}\left|T_{\nu \alpha k, \sigma p}\right|^{2} \delta\left(\epsilon_{L p}+e V-E_{\nu k}\right)\left(\epsilon_{L p}-\mu\right) \\
& \times\left[f\left(\epsilon_{L p}\right)-f\left(E_{\nu k}\right)\right] .
\end{aligned}
$$

If $P>0$ then the tunneling current cools the electron gas in the normal metal electrode. In order to determine $I$ and $P$ it is necessary to obtain $T_{\nu \alpha k, \sigma p}$ and $E_{\nu}(\mathbf{k})$. This is a nontrivial task for $\mathrm{HF}$ compounds due to the strong electron correlations. The physical properties of HF compounds depend strongly on temperature due to the Kondo effect. At temperatures $T \gg T_{k}$ bare electron states are well-defined quasiparticle states. With decreasing temperature when $T$ $\rightarrow T_{k}$, the scattering of conduction electrons off the localized $f$ electrons is enhanced and results in the Kondo resonance, which is responsible for the unusual transport properties of HF compounds. ${ }^{12}$ In particular, at $T \sim T_{k}$ the Seebeck coefficient of a HF metal displays a giant peak. At temperatures below $T_{k}$ hybrid quasiparticles are formed. They can be described as a quantum superposition of conduction and $f$ electrons. Thus, it is expected that tunneling of electrons from the normal metal electrode into the HF electrode is different above and below $T_{k}$ due to the different nature of quasiparticle states.

In order to study the Peltier effect of the $\mathrm{NIN}_{\mathrm{HF}}$ junction at $T<T_{k}$, the following Hamiltonian was considered: $H$ $=H_{L}+H_{R}+H_{T}$, where $H_{L}$ and $H_{R}$ are the Hamiltonians of the normal and HF metals, respectively. $H_{T}$ is the tunneling Hamiltonian: 


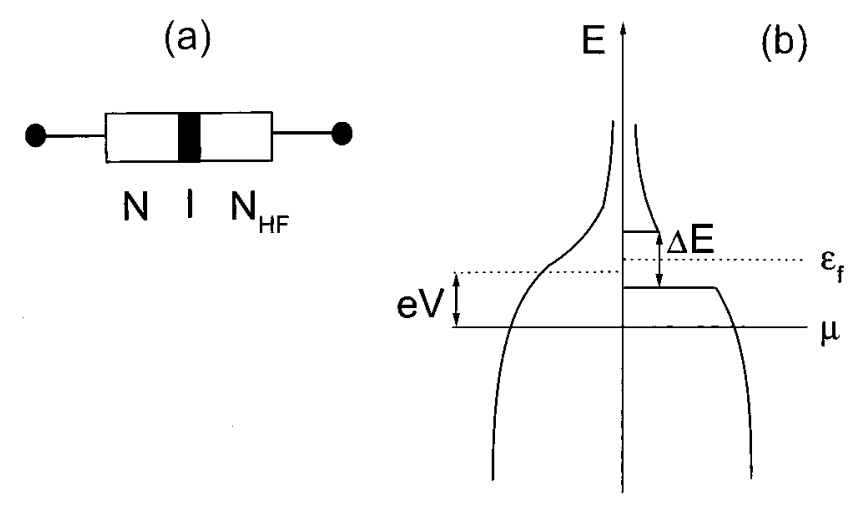

FIG. 1. (a) Schematic of the $\mathrm{NIN}_{\mathrm{HF}}$ junction. (b) Electron distribution function in the normal metal electrode (on the left) and HF electrode (on the right).

$$
H_{T}=\sum_{k, p, \sigma}\left(T_{k, p} a_{\sigma p}^{+} c_{\sigma k}+T_{k, p}^{*} c_{\sigma k}^{+} a_{\sigma p}\right)
$$

where $a_{\sigma p}^{+}\left(a_{\sigma p}\right)$ and $c_{\sigma k}^{+}\left(c_{\sigma k}\right)$ are creation (annihilation) operators for bare electron states with spin $\sigma$ and momentum $p$ and $k$ in the normal and HF metals, respectively, and $T_{k, p}$ is the bare matrix element. $H_{R}$ is the Hamiltonian of the periodic Anderson model. At $T<T_{k}$, in the framework of the mean-field approach, the Hamiltonian $H_{R}$ can be written in the form: ${ }^{13-15}$

$$
\begin{aligned}
H_{R}= & \sum_{\sigma k} \epsilon_{R k} c_{\sigma k}^{+} c_{\sigma k}+\sum_{\alpha k} \epsilon_{f} f_{a k}^{+} f_{a k} \\
& +\sum_{\sigma \alpha k}\left(V_{\mathrm{eff}} \phi_{\alpha \sigma}(\mathbf{k}) f_{a k}^{+} c_{\sigma k}+H . c .\right),
\end{aligned}
$$

where $\epsilon_{R k}$ is the kinetic energy of noninteracting conduction electrons in the HF metal, and $V_{\text {eff }}$ and $\epsilon_{f}$ are the effective hybridization parameter and effective $f$-level energy, respectively. The parameters $V_{\text {eff }}, \epsilon_{f}$ and the chemical potential $\mu$ have to be determined self-consistently, minimizing the free energy with respect to $V_{\text {eff }}, \epsilon_{f}$ and $\mu$ at a given total number $N_{t}$ of electrons. Here, $N_{t}=N_{c}+N_{f}$ where $N_{c}$ and $N_{f}$ are the number of conduction and $f$ electrons per $f$ ion, respectively. This gives a set of the mean-field equations. ${ }^{13} \mathrm{Ce}^{3+}$ ions in the ground state have one electron in the $f$ shell. Valence fluctuations lead to $N_{f} \lesssim 1$. In this case $\epsilon_{f}>\mu$. The function $\phi_{\alpha \sigma}(\mathbf{k})$ characterizes the anisotropy of the Kondo coupling. The diagonalization of the Hamiltonian (4) gives the quasiparticle energies

$$
E_{\nu k}=\left\{\epsilon_{f}+\epsilon_{R k} \mp\left[\left(\epsilon_{f}-\epsilon_{R k}\right)^{2}+4|V(\mathbf{k})|^{2}\right]^{1 / 2}\right\} / 2 .
$$

Here, $V(\mathbf{k}) \equiv V_{\text {eff }} \phi(\mathbf{k}), \phi^{2}(\mathbf{k}) \equiv \Sigma_{\alpha \sigma}\left|\phi_{\alpha \sigma}(\mathbf{k})\right|^{2} / 2$. The upper and lower signs correspond to $\nu=1$ and 2 , respectively. For the isotropic coupling $\phi^{2}(\mathbf{k})=1$ a hybridization gap between the lower and upper hybrid bands $E_{1 k}$ and $E_{2 k}$ is equal to $\Delta E \equiv \min E_{2}-\max E_{1} \approx W\left|V_{\mathrm{eff}}\right|^{2} /(W-\mu) \mu$, where $W$ is the width of the conduction band. The gap is opened at $\epsilon_{f}$. Calculating the eigenfunctions related to $E_{\nu k}$, the matrix element $T_{\nu \alpha k, \sigma p}$ can be found:

$$
T_{\nu \alpha k, \sigma p} \equiv T_{\alpha k, \sigma p} u_{\nu k}=i \phi_{\alpha \sigma}(\mathbf{k}) u_{\nu k} T_{k, p} / \phi(\mathbf{k}) .
$$

Equation (6) shows that $T_{\nu \alpha k, \sigma p}$ differs strongly from the bare matrix element $T_{k, p}$. The factors $i \phi_{\alpha \sigma}(\mathbf{k}) / \phi(\mathbf{k})$ and $u_{\nu k}$ describe the renormalization of the tunneling matrix element due to the anisotropy of the Kondo coupling and the formation of the hybrid states (5). There is the relation $\left|u_{\nu k}\right|^{2}=\partial E_{\nu k} / \partial \epsilon_{R k}$. The hybrid quasiparticles are called "heavy fermions" as the mass of the quasiparticles at the Fermi surface is much larger than the bare electron mass $m_{R}: m_{R}^{*} / m_{R}=\left|u_{1 k}\right|^{-2} \gg 1$ at $k=k_{F}$. In accordance with Eq. (6) at $T<T_{k}$ the renormalized matrix element $T_{\nu \alpha k, \sigma p}$ for tunneling into HF states near the Fermi surface becomes in $1 /\left|u_{1 k}\right| \sim\left(m_{R}^{*} / m_{R}\right)^{1 / 2}$ times smaller than the $T_{k, p}$ value. Substituting Eq. (6) into Eqs. (1) and (2) gives

$$
\begin{aligned}
I= & 4 \pi e \sum_{\nu k, p}\left|T_{k, p}\right|^{2}\left|u_{\nu k}\right|^{2} \delta\left(\epsilon_{L p}+e V-E_{\nu k}\right) \\
& \times\left[f\left(E_{\nu k}-e V\right)-f\left(E_{\nu k}\right)\right], \\
P= & 4 \pi \sum_{\nu k, p}\left|T_{k, p}\right|^{2}\left|u_{\nu k}\right|^{2} \delta\left(\epsilon_{L p}+e V-E_{\nu k}\right) \\
& \times\left(E_{\nu k}-e V-\mu\right)\left[f\left(E_{\nu k}-e V\right)-f\left(E_{\nu k}\right)\right] .
\end{aligned}
$$

It is important to note that the increase of the density of states (DOS) in the hybrid bands, $\rho_{\nu}(E) \equiv \Sigma_{k} \delta\left(E-E_{\nu k}\right)$ $=\rho_{R}\left|u_{\nu k}\right|^{-2}$, with regard to the bare DOS $\rho_{R}$, compensates for the decrease of the tunneling matrix element Eq. (6), as $\rho_{\nu}(E)\left|T_{\nu \alpha k, \sigma p}\right|^{2}=\rho_{R}\left|T_{k, p}\right|^{2}$. Taking into account this result, it is concluded that the formation of heavy fermions does not change noticeably the magnitude of the tunneling current $I$ through the $\mathrm{NIN}_{\mathrm{HF}}$ junction.

In order to examine the properties of the $\mathrm{NIN}_{\mathrm{HF}}$ junction caused by the formation of the hybridization gap, the following approximation is made. The energy dependences of the bare density of states is neglected, as is also the momentum dependence of $T_{k, p}$ and anisotropy of the Kondo coupling. With these assumptions the following is obtained:

$$
\begin{aligned}
I=4 & \pi e \rho_{L} \rho_{R}\left|T_{k, p}\right|^{2} \sum_{\nu} \int_{\min E_{\nu}}^{\max E_{\nu}}[f(E-e V)-f(E)] d E, \\
P= & 4 \pi \rho_{L} \rho_{R}\left|T_{k, p}\right|^{2} \sum_{\nu} \int_{\min E_{\nu}}^{\max E_{\nu}}(E-e V-\mu) \\
& \times[f(E-e V)-f(E)] d E .
\end{aligned}
$$

At $|e V| \ll T$ these equations give $P=\Pi I$ where

$$
\begin{aligned}
\Pi= & \left\{\Delta E+E_{t} f\left(E_{t}\right)-E_{b} f\left(E_{b}\right)-T \ln \left[f\left(E_{t}\right) / f\left(E_{b}\right)\right]\right\} / \\
& {\left[1+f\left(E_{b}\right)-f\left(E_{t}\right)\right]|e| . }
\end{aligned}
$$

Here, $E_{t}=\max E_{1 k}$ and $E_{b}=\max E_{2 k}$.

In order to obtain the temperature dependence of the Peltier coefficient $\Pi$ the set of mean-field equations are solved numerically and $\epsilon_{f}(T), V_{\text {eff }}(T)$, and $\mu(T)$ are obtained. These parameters determine the energy spectrum Eq. (5). The temperature dependences of $\Pi(T)$ and the energy gap $\Delta E$ are presented in Fig. 2. These calculations show that in the case of a low-lying Kramers doublet $N=2$ the Peltier coefficient $\Pi(T)$ achieves a peak value of order of $0.5 T_{k} /|e|$ at $T \propto 0.2 T_{k}$. Therefore, if $e V>0$, then a current flowing from the left to the right cools the electrons in the normal metal electrode. The cooling effect has the following origin. Consider a flow of electrons from the HF electrode into the normal metal electrode. Such a flow corresponds to $I>0$. In license or copyright; see http://apl.aip.org/about/rights_and_permissions 

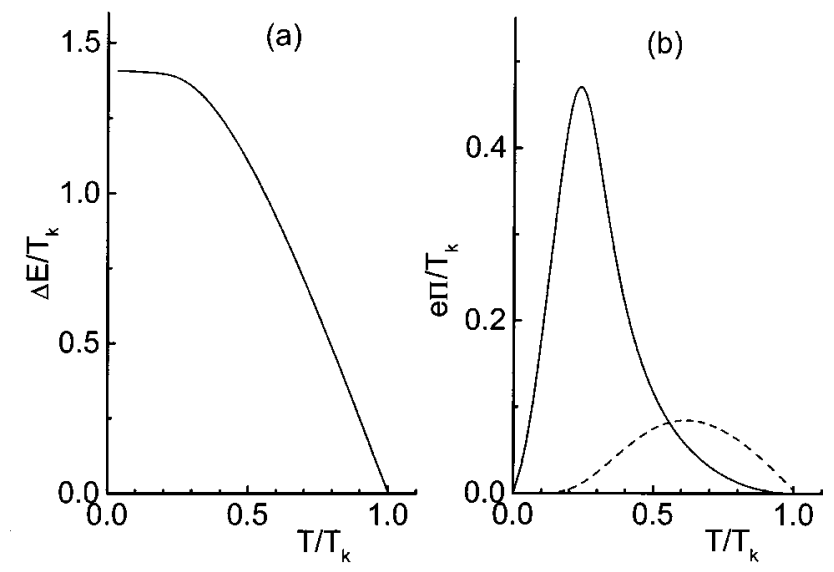

FIG. 2. (a) Hybridization gap $\Delta E$ vs $T$ in the HF metal for the spin degeneracy $N=2$ and concentration of conduction electrons $N_{c}=0.95$ per $f$ ion. (b) Temperature behavior of the Peltier coefficient $\Pi$ for the $\mathrm{NIN}_{\mathrm{HF}}$ junction determined from Eq. (10) at $N=2$ (solid line) and $N=8$ (dashed line).

accordance with the above calculations, electrons with an average energy smaller than $\mu$ are transferred from the HF electrode into the normal metal due to the hybridization gap which lies above the chemical potential $\mu$ in the energy spectrum of the HF metal, while electrons with an average energy equal to $\mu$ leave the normal metal electrode through normal contact.

In the case of impurity scattering at $T<T_{k}$ the Peltier coefficient $\Pi_{\mathrm{HF}}$ of $\mathrm{HF}$ metals is equal to $\Pi_{\mathrm{HF}}$ $=\pi^{2} T^{2} N_{f} /\left(3 e N_{c} T_{0}\right)$ where $T_{0}$ is the low temperature Kondo scale and $T_{0} \propto T_{k} .{ }^{16}$ In accordance with the calculations represented in Fig. 2 for $N=2$, at $T=T_{m}$, the ratio of the peak value $\Pi\left(T_{m}\right)$ to $\Pi_{\mathrm{HF}}$ is given by $\Pi\left(T_{m}\right) / \Pi_{\mathrm{HF}}$ $\approx 0.15 N_{c} T_{k}^{2} / N_{f} T_{m}^{2}$. As $N_{c} \sim N_{f}$ and $T_{m} \approx 0.24 T_{k}, \Pi\left(T_{m}\right)$ is three times larger than $\Pi_{\mathrm{HF}}$. This means that at low temperatures $T \sim T_{m}$ the $\mathrm{NIN}_{\mathrm{HF}}$ junction is substantially more efficient in cooling than the normal metal-heavy fermion metal contact.

Unlike $\mathrm{Ce}^{3+}$ ions, a magnetic moment of $\mathrm{Yb}^{3+}$ ions is produced by one hole in its $f$ shell, and the hybridization gap is opened at $\epsilon_{f}<\mu$. If the right electrode is made from a Yb-based HF compound, then $\Pi$ is negative unlike the positive $\Pi$ for Ce-based HF compounds. In the latter case, electrons in the normal metal thermoelement are cooled when $e V<0$. For more effective cooling of the normal metal elec- trode a junction $\mathrm{N}_{\mathrm{HF}}(\mathrm{Yb}) \mathrm{ININ}_{\mathrm{HF}}(\mathrm{Ce})$ may be proposed because the current flowing through both $\mathrm{N}_{\mathrm{HF}}(\mathrm{Yb}) \mathrm{IN}$ and $\mathrm{NIN}_{\mathrm{HF}}(\mathrm{Ce})$ junctions will cool the electrons in the normal metal electrode.

The above calculations of the Peltier coefficient of $\mathrm{NIN}_{\mathrm{HF}}$ junctions have been carried out in the isotropic approximation neglecting anisotropy of the Kondo coupling produced by the crystal field effect. It is well known that this effect influences the DOS in HF compounds. ${ }^{14,15}$ It is also expected that it will affect the tunneling current and the power transfer through the junction.

In conclusion, the tunneling current and Peltier effect of the normal metal-insulator-heavy fermion metal junctions have been studied. It has been demonstrated that at temperatures below the Kondo temperature the tunneling current transfers energy from the normal metal cooling electrons in the normal metal electrode. Thus, these junctions as well as the normal-insulator-superconductor junctions may be used as an electronic microrefrigerator to cool electrons in the normal metal electrode below the lattice temperature.

This work was supported by the EPSRC (U.K.) Grant No. 04003/01 and in part by the Russian Fund for Basic Research, Grant No. 01-02-17794.

${ }^{1}$ N. Grewe and F. Steglich, in Handbook on the Physics and Chemistry of Rare Earths, Vol. 14, edited by K. A. Gschneidner, Jr., and L. Eyring (North-Holland, Amsterdam, 1991), p. 343.

${ }^{2}$ A. C. Hewson, The Kondo Problem to Heavy Fermions (Cambridge University Press, Cambridge, U.K., 1993).

${ }^{3}$ G. D. Mahan, B. Sales, and J. Sharp, Phys. Today 50, 42 (1997); G. D. Mahan, Solid State Phys. 51, 81 (1997).

${ }^{4}$ W. Mao and K. S. Bedell, Phys. Rev. B 59, R15590 (1999).

${ }^{5}$ M. Rontani and R. J. Sham, Appl. Phys. Lett. 77, 3033 (2000).

${ }^{6}$ M. Nahum, T. M. Eiles, and J. M. Martinis, Appl. Phys. Lett. 65, 3123 (1994).

${ }^{7}$ A. Bardas and D. Averin, Phys. Rev. B 52, 12873 (1995).

${ }^{8}$ N. M. Miskovsky and P. H. Cutler, Appl. Phys. Lett. 75, 2147 (1999).

${ }^{9}$ Y. Hishinuma, T. H. Geballe, B. Y. Moyzhes, and T. W. Kenny, Appl. Phys. Lett. 78, 2572 (2001)

${ }^{10}$ T. S. Fisher, Appl. Phys. Lett. 79, 3699 (2001).

${ }^{11}$ A. M. Savin, M. Prinnila, P. P. Kivinen, J. P. Pekola, J. Ahopelto, and A. J. Manninen, Appl. Phys. Lett. 79, 1471 (2001).

${ }^{12}$ N. E. Bickers, D. L. Cox, and J. W. Wilkins, Phys. Rev. B 36, 2036 (1987).

${ }^{13}$ D. M. Newns and N. Read, Adv. Phys. 36, 799 (1987).

${ }^{14}$ H. Ikeda and K. Miyake, J. Phys. Soc. Jpn. 65, 1769 (1996)

${ }^{15}$ J. Moreno and P. Coleman, Phys. Rev. Lett. 84, 342 (2000).

${ }^{16}$ V. I. Belitsky and A. V. Goltsev, Physica B 172, 459 (1991). 